\title{
Iron(III) Catalyzed Coupling of Aryl Halides: Synthesis of Terphenyl and Biphenyl Derivatives
}

\author{
M. J. Rahman*, R. Pervin, R. Hasan, and D. Debnath \\ Department of Chemistry, Shahjalal University of Science and Technology, Sylhet 3114, \\ Bangladesh
}

Received 16 October 2012, accepted in final revised form 5 December 2012

\begin{abstract}
Iron(III) mediated coupling of arylmagnesium halides with other aryl halides produced both homo- and cross-coupled products. The reaction of phenylmagnesium bromide with 1,4dibromobenzene as well as with 4-chlorobromobenzene in presence of $\mathrm{FeCl}_{3}$ catalyst produced both cross-coupled $p$-terphenyl and homo-coupled biphenyl. Under the same condition only homo-coupled 4,4'-dipropoxybiphenyl was obtained from 4propoxyphenylmagnesium bromide. The coupling of 4-bromophenylmagnesium bromide with bromobenzene produced both homo- and cross-coupled biaryls.
\end{abstract}

Keywords: Coupling; Aryl halides; Terphenyl; Biphenyl.

@ 2013 JSR Publications. ISSN: 2070-0237 (Print); 2070-0245 (Online). All rights reserved.

doi: http://dx.doi.org/10.3329/jsr.v5i1.12207 J. Sci. Res. 5 (1), 127-134 (2013)

\section{Introduction}

Aryl-aryl bond formation is one of the most powerful tools in modern organic synthesis [1-4]. Biaryls as well as their heteroaromatic analogues are some of the attractive structural units in natural products, bio-active compounds, functional polymers, ligands in catalysts etc. For the synthesis of biaryls, transition-metal-mediated coupling reactions have been used in most cases. Palladium and nickel catalysts have been the major choice for aryl-aryl cross-coupling reactions and are widely used in the laboratory as well as in industry. Palladium(0) catalysts are reliably used [5-8], especially if appropriate ligands such as sterically hindered phosphines are present [9-12]. Nickel(0) complexes had also found useful applications, but appear to have less general scope [13-17].

Following the pioneering work of Kochi and co-workers [18-22] iron catalysts have recently been very actively investigated for their performance in cross-coupling reactions

*Corresponding author: mjrche@yahoo.com 
[23-29]. Because of competition of homo-coupling over cross-coupling, there remained a challenge of using iron-catalyst for aryl-aryl cross-coupling. Although highly efficient cross-coupling reactions could be realized between a range of alkyl magnesium reagents and aryl halides or aryl sulfonates, iron-catalyzed cross-coupling between two aryl moieties remained problematic owing to extensive homo-coupling reactions of the aryl magnesium species. Nakamura et al. reported a selective biaryl synthesis based on iron(III) fluoride catalyzed cross-coupling of aryl chlorides with aryl Grignard reagents where the fluoride ion remarkably suppresses the homocoupling in presence of additive like 1,3-bis(2,6-diisopropylphenyl)imidazolinium chloride (SIPr.HCl) [30]. Very recently Knokhel and co-workers reported iron(III) bromide mediated coupling of N-heterocyclic chlorides and bromides with arylmagnesium bromide lithium chloride complex [31]. Herein, we report the formation of cross-coupled biaryls along with homo-coupled products through iron(III) chloride catalyzed reactions of aryl Grignard reagents and aryl halides without using any additives.

\section{Experimental}

\subsection{General}

Melting points were determined with Yanaco MP-500D melting point apparatus. NMR spectra were recorded on Bruker Biospin 500 spectrometer using tetramethylsilane as the internal standard. Mass spectra were recorded on a Shimadzu GCMS-QP2010 spectrometer. All solvents were dried and purified by the usual techniques: tetrahydrofuran was distilled from benzophenone ketyl under a nitrogen atmosphere; $n$ hexane and dichloromethane were simply distilled without using any drying agent.

\subsection{General Procedure for the cross-coupling of arylmagnesium bromide and aryl halide (typical for coupling of phenylmagnesium bromide with 1,4-dibromobenzene)}

A solution of bromobenzene $(785 \mathrm{mg}, 5.0 \mathrm{mmol})$ in dry THF $(3 \mathrm{~mL})$ was added dropwise to a portion of $\mathrm{Mg}$ turnings $(132 \mathrm{mg}, 5.5 \mathrm{mmol})$ in dry THF $(3 \mathrm{~mL})$ under a nitrogen atmosphere at room temperature. Exothermic reaction started within few minutes. The reaction mixture was stirred at room temperature for $1.5 \mathrm{~h}$ to complete the formation of Grignard reagent. With the help of a syringe the Grignard reagent thus formed was transferred to a $50 \mathrm{~mL}$ two-necked round-bottomed flask under nitrogen atmosphere. A 5 $\mathrm{mL}$ portion dry THF was added to dilute the Grignard reagent and then it was cooled to $0{ }^{\circ} \mathrm{C}$. Then anhydrous $\mathrm{FeCl}_{3}$ (41 mg, $5 \mathrm{~mol} \%$ ) and 1,4-dibromobenzene (590 mg, 2.5 $\mathrm{mmol}$ ) were added to the reaction mixture and was allowed to stand at room temperature. It was then stirred overnight to complete the reaction. Methanol $(10 \mathrm{~mL})$ was added followed by the addition of $\mathrm{CH}_{2} \mathrm{Cl}_{2}(20 \mathrm{~mL})$ and water. The organic layer was separated and the aqueous layer was extracted with more $\mathrm{CH}_{2} \mathrm{Cl}_{2}$. The combined organic layer was dried over anhydrous $\mathrm{MgSO}_{4}$ and the solvent was removed in a vacuum rotary evaporator 
to give a crude product. The crude was chromatographed on a silica gel column eluting with hexane to afford $218 \mathrm{mg}$ (38\%) of $p$-terphenyl as cross-coupled product and $65 \mathrm{mg}$ (17\%) of biphenyl as homo-coupled product.

p-Terphenyl (5): white solid, $\mathrm{mp} 211.0-212.0^{\circ} \mathrm{C}$ (lit [32] $\mathrm{mp} 210-211.5^{\circ} \mathrm{C}$ ); EI MS: $\mathrm{m} / \mathrm{z} 230\left(\mathrm{M}^{+}\right) ;{ }^{1} \mathrm{H}$ NMR $\left(\mathrm{CDCl}_{3}, 500 \mathrm{MHz}\right): \delta(\mathrm{ppm}) 7.68(\mathrm{~s}, 4 \mathrm{H}), 7.65(\mathrm{~d}, J=7.8 \mathrm{~Hz}$, $4 \mathrm{H}), 7.45(\mathrm{t}, J=7.8 \mathrm{~Hz}, 4 \mathrm{H}), 7.36(\mathrm{t}, J=7.6 \mathrm{~Hz}, 2 \mathrm{H}) ;{ }^{13} \mathrm{C} \mathrm{NMR}\left(\mathrm{CDCl}_{3}, 125 \mathrm{MHz}\right): \delta$ (ppm) 140.7, 140.1, 128.8, 127.5, 127.3, 127.0.

Biphenyl (6): Colorless crystal, mp 69.0-70.0 ${ }^{\circ} \mathrm{C}$ (lit [33] mp 69.0-69.5 ${ }^{\circ} \mathrm{C}$ ); EI MS $\mathrm{m} / \mathrm{z} 154\left(\mathrm{M}^{+}\right) ;{ }^{1} \mathrm{H}$ NMR $\left(\mathrm{CDCl}_{3}, 500 \mathrm{MHz}\right): \delta(\mathrm{ppm}) 7.61(\mathrm{~d}, J=8.4 \mathrm{~Hz}, 4 \mathrm{H}), 7.45(\mathrm{t}, J=$ $7.8 \mathrm{~Hz}, 4 \mathrm{H}), 7.36(\mathrm{t}, J=7.4 \mathrm{~Hz}, 2 \mathrm{H}) ;{ }^{13} \mathrm{C} \mathrm{NMR}\left(\mathrm{CDCl}_{3}, 125 \mathrm{MHz}\right): \delta(\mathrm{ppm}) 141.2,128.7$, 127.2, 127.1 .

\subsubsection{Coupling of 4-bromophenylmagnesium bromide with bromobenzene}

Starting materials and reagents used were 1,4-dibromobenzene (1.180 g, $5.0 \mathrm{mmol}), \mathrm{Mg}$ turnings (134 mg, $5.6 \mathrm{mmol}), \mathrm{FeCl}_{3}$ (40 mg, $5 \mathrm{~mol} \%$ ) and bromobenzene (786 mg, 5.0 mmol). Products were 4,4'-dibromobiphenyl (47 mg, 8\%), 4-bromobiphenyl (186 mg, $16 \%)$ and $p$-terphenyl (34 mg, 3\%).

4,4'-Dibromobiphenyl (8): Colorless crystal, mp 165.0-166. $0{ }^{\circ} \mathrm{C}$ (lit [34] mp 166.5167.0 $\left.{ }^{\circ} \mathrm{C}\right)$; EI MS: m/z 310/312/314 (1:2:1) $\left(\mathrm{M}^{+}\right) ;{ }^{1} \mathrm{H}$ NMR $\left(\mathrm{CDCl}_{3}, 500 \mathrm{MHz}\right): \delta(\mathrm{ppm})$ $7.56(\mathrm{~d}, J=8.4 \mathrm{~Hz}, 4 \mathrm{H}), 7.41(\mathrm{~d}, J=8.4 \mathrm{~Hz}, 4 \mathrm{H}) ;{ }^{13} \mathrm{C} \mathrm{NMR}\left(\mathrm{CDCl}_{3}, 125 \mathrm{MHz}\right): \delta(\mathrm{ppm})$ $138.9,132,128.5,121.9$.

4-Bromobiphenyl (7): White solid, $\mathrm{mp} 88.0-89.0^{\circ} \mathrm{C}$ (lit [35] $\mathrm{mp} 89.0-90.0^{\circ} \mathrm{C}$ ); EI MS: $\mathrm{m} / \mathrm{z} 232 / 234(1: 1)\left(\mathrm{M}^{+}\right) ;{ }^{1} \mathrm{H} \mathrm{NMR}\left(\mathrm{CDCl}_{3}, 500 \mathrm{MHz}\right): \delta(\mathrm{ppm}) 7.60-7.51(\mathrm{~m}, 4 \mathrm{H})$, 7.48-7.36 (m, 4H), $7.35(\mathrm{t}, J=7.6 \mathrm{~Hz}, 1 \mathrm{H}) ;{ }^{13} \mathrm{C} \mathrm{NMR}\left(\mathrm{CDCl}_{3}, 125 \mathrm{MHz}\right): \delta(\mathrm{ppm}) 140.2$, 140.0, 131.9, 128.9, 128.8, 127.6, 127.0, 121.6.

p-Terphenyl (5): white solid, $\mathrm{mp} 211.0-212.0{ }^{\circ} \mathrm{C}$ (lit [32] mp 210-211. $5^{\circ} \mathrm{C}$ ); EI MS: $\mathrm{m} / \mathrm{z} 230\left(\mathrm{M}^{+}\right) ;{ }^{1} \mathrm{H} \mathrm{NMR}\left(\mathrm{CDCl}_{3}, 500 \mathrm{MHz}\right): \delta(\mathrm{ppm}) 7.68(\mathrm{~s}, 4 \mathrm{H}), 7.65(\mathrm{~d}, J=7.8 \mathrm{~Hz}$, $4 \mathrm{H}), 7.45(\mathrm{t}, J=7.8 \mathrm{~Hz}, 4 \mathrm{H}), 7.36(\mathrm{t}, J=7.6 \mathrm{~Hz}, 2 \mathrm{H}) ;{ }^{13} \mathrm{C} \mathrm{NMR}\left(\mathrm{CDCl}_{3}, 125 \mathrm{MHz}\right): \delta$ (ppm) 140.7, 140.1, 128.8, 127.5, 127.3, 127.0.

\subsubsection{Coupling of 4-propoxyphenylmagnesium bromide with 1,4-dibromobenzene}

Starting materials and reagents used were 4-propoxybromobenzene (1.075 g, $5.0 \mathrm{mmol})$, Mg turnings (132 mg, $5.5 \mathrm{mmol}$ ), $\mathrm{FeCl}_{3}$ (40 mg, $5 \mathrm{~mol} \%$ ) and 1,4-dibromobenzene (592 $\mathrm{mg}, 2.5 \mathrm{mmol}$ ). Product is 4,4'-dipropoxybiphenyl (492 mg, 73\%).

4,4'Dipropoxybiphenyl (9): White solid, mp 146-147 ${ }^{\circ} \mathrm{C}$, EI MS: $\mathrm{m} / \mathrm{z} 270\left(\mathrm{M}^{+}\right) ;{ }^{1} \mathrm{H}$ NMR $\left(\mathrm{CDCl}_{3}, 500 \mathrm{MHz}\right): \delta(\mathrm{ppm}) 7.46(\mathrm{~d}, J=8.8 \mathrm{~Hz}, 4 \mathrm{H}), 6.94(\mathrm{~d}, J=8.8 \mathrm{~Hz}, 4 \mathrm{H}), 3.95$ (t, $J=6.6 \mathrm{~Hz}, 2 \mathrm{H}$ ), 1.82 (sextet, $J=7.0 \mathrm{~Hz}, 2 \mathrm{H}$ ), $1.05\left(\mathrm{t}, J=7.3 \mathrm{~Hz}, 3 \mathrm{H}\right.$ ); ${ }^{13} \mathrm{C} \mathrm{NMR}$ $\left(\mathrm{CDCl}_{3}, 125 \mathrm{MHz}\right): \delta(\mathrm{ppm}) 158.2,133.3,127.6,114.7,69.6,22.6,10.5$. 


\subsubsection{Coupling of phenylmagnesium bromide with 4-chlorobromobenzene}

Starting materials and reagents used were bromobenzene $(786 \mathrm{mg}, 5.0 \mathrm{mmol}), \mathrm{Mg}$ turnings (130 mg, $5.4 \mathrm{mmol}$ ), $\mathrm{FeCl}_{3}(42 \mathrm{mg}, 5 \mathrm{~mol} \%$ ) 1-bromo-4-chlorobenzene (480 mg, $2.5 \mathrm{mmol}$ ) Products were $p$-terphenyl (58 mg, 10\%), biphenyl (181 mg, 47\%).

p-Terphenyl (5): white solid, mp 211.0-212.0 ${ }^{\circ} \mathrm{C}$ (lit [32] mp 210-211.5 ${ }^{\circ} \mathrm{C}$ ); EI MS: $\mathrm{m} / \mathrm{z} 230\left(\mathrm{M}^{+}\right) ;{ }^{1} \mathrm{H} \mathrm{NMR}\left(\mathrm{CDCl}_{3}, 500 \mathrm{MHz}\right): \delta(\mathrm{ppm}) 7.68(\mathrm{~s}, 4 \mathrm{H}), 7.65(\mathrm{~d}, J=7.8 \mathrm{~Hz}$, $4 \mathrm{H}), 7.45(\mathrm{t}, J=7.8 \mathrm{~Hz}, 4 \mathrm{H}), 7.36(\mathrm{t}, J=7.6 \mathrm{~Hz}, 2 \mathrm{H}) ;{ }^{13} \mathrm{C} \mathrm{NMR}\left(\mathrm{CDCl}_{3}, 125 \mathrm{MHz}\right): \delta$ (ppm) 140.7, 140.1, 128.8, 127.5, 127.3, 127.0.

Biphenyl (6): Colorless crystal, mp 69.0-70.0 ${ }^{\circ} \mathrm{C}$ (lit [33] mp 69.0-69.5 ${ }^{\circ} \mathrm{C}$ ); m/z 154 $\left(\mathrm{M}^{+}\right) ;{ }^{1} \mathrm{H} \mathrm{NMR}\left(\mathrm{CDCl}_{3}, 500 \mathrm{MHz}\right): \delta(\mathrm{ppm}) 7.61(\mathrm{~d}, J=8.4 \mathrm{~Hz}, 4 \mathrm{H}), 7.45(\mathrm{t}, J=7.8 \mathrm{~Hz}$, $4 \mathrm{H}), 7.36(\mathrm{t}, J=7.4 \mathrm{~Hz}, 2 \mathrm{H}) ;{ }^{13} \mathrm{C} \mathrm{NMR}\left(\mathrm{CDCl}_{3}, 125 \mathrm{MHz}\right): \delta(\mathrm{ppm}) 141.2,128.7,127.2$, 127.1 .

\section{Results and Discussion}

Iron-catalysts being environmentally friendly and inexpensive have been extensively studied for the carbon-carbon bond formation. Iron(III) catalysts are capable of forming ferrate complex possessing excess aryl groups with appropriate reagents. The reductive elimination of these complexes might lead to the formation of both homo- and or crosscoupled products under suitable conditions.

In the present study, cross-coupled biaryls are produced along with homo-coupled products during reactions between aryl Grignard reagent and aryl halides in presence of $\mathrm{Fe}(\mathrm{III})$ catalyst. All Grignard reagents were prepared from pure aryl bromides and activated Mg turnings in dry THF under nitrogen atmosphere. The Grignard reagents so produced were diluted with dry THF before addition of another aryl halide and $\mathrm{FeCl}_{3}$ catalyst $(5 \mathrm{~mol} \%)$. The results of the coupling reactions are summarized in the Table 1.

The reaction of phenylmagnesium bromide with half-equivalent ( 0.5 equiv.) of 1,4dibromobenzene $\mathbf{2}$ in presence of Fe(III) catalyst as well as in the absence of any additive produced the cross-coupled product $p$-terphenyl $\mathbf{5}$ as the major product along with homocoupled product biphenyl 6. However the Grignard reagent prepared from 1,4dibromobenzene 2 and $\mathrm{Mg}$ turnings (1.1 equiv.), when coupled with bromobenzene 1 (1.0 equiv.), produced cross-coupled 4-bromobiphenyl 7 (16\%), p-terphenyl 5 (3\%) along with homo-coupled 4,4'-dibromobiphenyl 8 (8\%). In this case, the Grignard reagent 4bromophenylmagnesium bromide, which already contains a bromine atom in its para position, might produce mixture of $p$-oligophenyl derivatives under the reaction condition. The formation $p$-terphenyl $\mathbf{5}$ in this case can be explained by the coupling of di-Grignard derivative of 1,4-dibromobenzene (formed from excess $\mathrm{Mg}$ turnings) with bromobenzene 1. The Grignard reagent from 4-propoxybromobenzene 3, when treated with 1,4dibromobenzene 2 ( 0.5 equiv.) in presence of iron catalyst produced only homo-coupled 4,4'-dipropoxybiphenyl 9 (73\%). In this case, probably the electron donating alkoxy group 
in para position might accelerate the formation of ferrate complexes containing excess 4propoxyphenyl group (such as $\mathrm{Ar}_{4}^{1} \mathrm{Fe}, \mathrm{Ar}_{3}{ }_{3} \mathrm{Ar}^{2} \mathrm{Fe}$ and $\mathrm{Ar}_{2}^{1} \mathrm{Ar}{ }^{2} \mathrm{Fe}$ ), which undergo reductive elimination to produce only the homo-coupled product. However, the coupling between phenylmagnesium bromide and 4-chlorobromobenzene 4 ( 0.5 equiv.) produced only $10 \%$ of the expected cross-coupled product $p$-terphenyl $\mathbf{5}$ along with homo-coupled biphenyl $6(47 \%)$.

Table 1. Coupling of aryl halides using iron(III) catalyst.

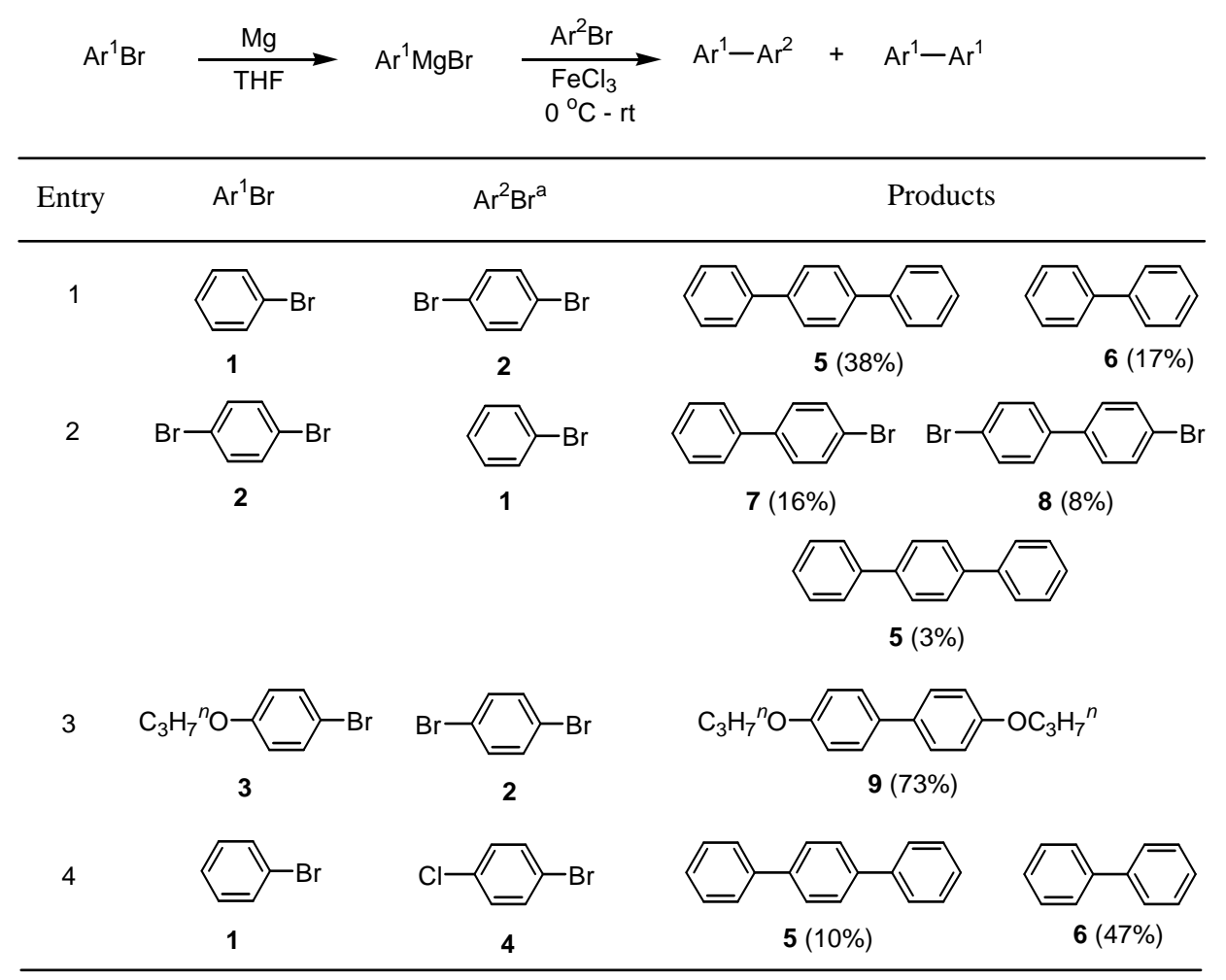

a In entry 1, 3 and 4 dihalobenzenes were used 0.5 equivalent in each case.

In a plausible catalytic cycle (Scheme 1) an addition of $\mathrm{Fe}(\mathrm{III})$ to an arylmagnesium halide to form a tetrakis(aryl)iron(III) intermediate is involved. This then undergoes reduction, losing two aryl groups as the homo-coupled product, and forms aryliron $(0)$. Addition of one equivalent arylmagnesium halide forms a low-valent, $\mathrm{Fe}^{0}$ species which serves as a reductant for the other aryl halide substrate. This forms an aryl radical and an open-shell $\mathrm{Fe}^{\mathrm{I}}$ radical which rapidly combines to create a tris(aryl)iron(II) species having two different kinds of aryl moieties. This then undergoes reductive elimination to yield the cross-coupled product and regenerate the active catalyst complex. 


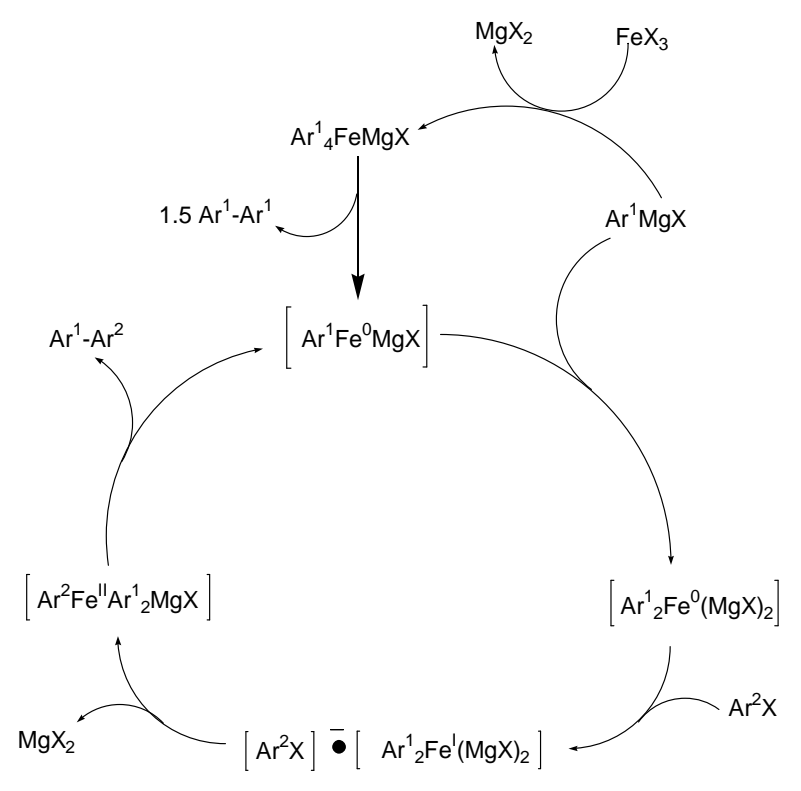

Scheme 1. Plausible mechanism for the iron(III) catalyzed coupling reaction

\section{Conclusion}

In summary, iron(III) chloride catalyzed coupling of aryl Grignard reagents with aryl halides produced both homo- and cross-coupled products. Coupling of phenylmagnesium bromide with half-equivalent of either 1,4-dibromobenzene or 1-bromo-4-chlorobenzene in presence of iron(III) catalyst produced both cross-coupled $p$-terphenyl and homocoupled biphenyl. Only homo-coupled 4,4'-dipropoxybiphenyl was obtained from 4propoxyphenylmagnesium bromide under the same condition. In this case, electrondonating propoxy group in para position might accelerate the formation of ferrate complexes containing excess aryl group that may undergo reductive elimination to produce only the homo-coupled product. The Grignard reagent prepared from 1,4dibromobenzene and $\mathrm{Mg}$ turnings (1.1 equiv.) when coupled with bromobenzene produced cross-coupled 4-bromobiphenyl and $p$-terphenyl along with homo-coupled 4,4'dibromobiphenyl.

\section{Acknowledgment}

We would like to thank Dr. Eigo Isomura (Deapartment of Chemistry, Tokyo Metropolitan University, Japan) for the spectral measurements such as ${ }^{1} \mathrm{H}$ NMR, ${ }^{13} \mathrm{C}$ NMR, and GS-MS. 


\section{References}

1. J. Hassan, M. Sévignon, C. Gozzi, E. Schulz, and M. Lemaire, Chem. Rev. 102, 1359 (2002). http://dx.doi.org/10.1021/cr000664r

2. G. Bringmann, R. Walter, and R. Weirich, Angew. Chem., Int. Ed. Engl. 29, 977 (1990). http://dx.doi.org/10.1002/anie.199009771

3. M. Sainsbury, Tetrahedron 36, 3327 (1980). http://dx.doi.org/10.1016/0040-4020(80)80185-2

4. G. Bringmann, A. J. P. Mortimer, P. A. Keller, M. J. Gresser, J. Garner, and M. Breuning, Angew. Chem. Int. Ed. 44, 5384 (2005). http://dx.doi.org/10.1002/anie.200462661

5. F. Diederich, and P. J. Stang, Metal-Catalysed Cross-Coupling Reactions, 1st Ed. (Wiley-VCH: Weinhein, Germany, 1998) p. 1-166. http://dx.doi.org/10.1002/9783527612222

6. J. Tsuji, Transition Metal Reagents and Catalysts: Innovations in Organic Synthesis, (Wiley, Chichester 1995) p. 27-108.

7. N. Miyaura, Cross-Coupling Reactions. A Practical Guide, Top. Curr. Chem., 219 (2002).

8. E. Negishi, Handbook of organopalladium Chemistry for Organic Synthesis, (WileyInterscience, New York, 2002) p. 39-188.

9. C. Dai, and G. C. Fu, J. Am. Chem. Soc. 123, 2719 (2001). http://dx.doi.org/10.1021/ja003954y

10. A. F. Littke, L. Schwarz, and G. C. Fu, J. Am. Chem. Soc. 124, 6343 (2002). http://dx.doi.org/10.1021/ja020012f

11. I. D. Hills, M. R. Netherton, and G. C. Fu, Angew. Chem. Int. Ed. 42, 5749 (2003). http://dx.doi.org/10.1002/anie.200352858

12. F. Rataboul, A. Zapf, R. Jackstell, S. Harkal, T. Riermeier, A. Monsees, U. Dingerdissen, and M. Beller, Chem. Eur. J. 10, 2983 (2004). http://dx.doi.org/10.1002/chem.200306026

13. T. -Y. Luh, Acc. Chem. Res. 24, 257 (1991). http://dx.doi.org/10.1021/ar00009a002

14. A. F. Indolese, Tetrahedron Lett. 38, 3513 (1997). http://dx.doi.org/10.1016/S0040-4039(97)00707-7

15. A. Sophia, E. Karlström, K. Itami, and J.-E. Bäckvall, J. Am. Chem. Soc. 122, 6950 (2000). http://dx.doi.org/10.1021/ja001440t

16. J. Montgomery, Acc. Chem. Res. 33, 467 (2000). http://dx.doi.org/10.1021/ar990095d

17. B. H. Lipshutz, Adv. Synth. Catal. 343, 313 (2001). http://dx.doi.org/10.1002/16154169(20010430)343:4<313::AID-ADSC313>3.0.CO;2-A

18. M. Tamura, and J. K. Kochi, J. Am. Chem. Soc. 93, 1487 (1971). http://dx.doi.org/10.1021/ja00735a030

19. M. Tamura, and J. K. Kochi, Synthesis 93, 303 (1971). http://dx.doi.org/10.1055/s-1971-35043

20. M. Tamura, and J. K. Kochi, J. Organomet. Chem. 31, 289 (1971). http://dx.doi.org/10.1016/S0022-328X(00)86239-7

21. J. K. Kochi, Acc. Chem. Res. 7, 351 (1974). http://dx.doi.org/10.1021/ar50082a006

22. R. S. Smith, and J. K. Kochi, J. Org. Chem. 41, 502 (1976). http://dx.doi.org/10.1021/jo00865a019

23. G. Cahiez, and S. Marquais, Pure Appl. Chem. 68, 669 (1996). http://dx.doi.org/10.1351/pac199668010053

24. G. Cahiez, and S. Marquais, Tetrahedron Lett. 37, 1773 (1996). http://dx.doi.org/10.1016/0040-4039(96)00116-5

25. G. Cahiez, H. Avedissian, Synthesis 8, 1199 (1998). http://dx.doi.org/10.1055/s-1998-2135

26. M. Hocek, and H. Dvoráková, J. Org. Chem. 68, 5773 (2003). http://dx.doi.org/10.1021/jo034351i

27. B. Hölzer, and R.W. Hoffmann, Chem. Commun. 6, 732 (2003). http://dx.doi.org/10.1039/b300033h

28. M. Hojo, Y. Murakami, H. Aihara, R. Sakuragi, Y. Baba, and A. Hosomi, Angew. Chem. Int. Ed. 40, 621 (2001). http://dx.doi.org/10.1002/1521-3773(20010202)40:3<621::AID-ANIE621>3.0.CO;2-O 


\section{Iron(III) Catalyzed Coupling}

29. M. Nakamura, A. Hirai, and E. Nakamura, J. Am. Chem. Soc. 123, 978 (2001). http://dx.doi.org/10.1021/ja003389z

30. T. Hatakeyama, and M. Nakamura, J. Am. Chem. Soc. 129, 9844 (2007). http://dx.doi.org/10.1021/ja0730841

31. O. M. Kuzmina, A. K. Steib, D. Flubacher, and P. Knochel, Org. Lett. 14, 4818 (2012). http://dx.doi.org/10.1021/ol302136c

32. R. T. Arnold, C. Collins, and W. Zenk, J. Am. Chem. Soc. 62, 983 (1940). http://dx.doi.org/10.1021/ja01861a501

33. L. W. Pickett, G. F. Walter, and H. France, J. Am. Chem. Soc. 58, 2296 (1936).

34. B. Williamson, and W. H. Rodebush, J. Am. Chem. Soc. 63, 3018 (1941). http://dx.doi.org/10.1021/ja01302a061

35. J.-F. Wei, J. Jiao. J.-J. Feng, J. Lv, X.-R. Zhang, X.-Y. Shi, and Z.-G. Chen, J. Org. Chem. 74, 6283 (2009). http://dx.doi.org/10.1021/jo900481y 